\title{
Responses of the antioxidative enzymes in Malaysian rice (Oryza sativa L.) cultivars under submergence condition
}

\begin{abstract}
The potential involvement of activated oxygen species by submergence stress was studied in two Malaysian rice cultivars, MR219-4 and MR219-9, and cultivar FR13A that is known to be tolerant to submergence. Seedlings of these three rice cultivars were subjected to different submergence periods ( 4,8 , and 12 days). Under 8 days of complete submergence, FR13A cultivar showed higher lipid peroxidation in terms of malondialdehyde level and activities of antioxidative enzymes, superoxide dismutase (SOD), catalase (CAT), ascorbate peroxidase (APX), and glutathione reductase (GR) when compared to the MR219-4 and MR219-9 cultivars. MR219-9 showed higher SOD, APX, and GR activities after 12 days of submergence. The levels of SOD activity indicated that detoxification of $\mathrm{O} 2$ - to $\mathrm{H} 2 \mathrm{O} 2$ was maintained at a stable level throughout the submergence stress until up to 8 days and increased rapidly at 12 days of submergence. The results indicated that tolerance to submergence in rice is associated until 8 days submergence for MR219-4 and FR13A cultivars. These findings suggested that tolerance to submergence stress in rice might be proven by increased the capacity of antioxidative system. In addition, CAT activity has much higher affinity for scavenges $\mathrm{H} 2 \mathrm{O} 2$ than APX. Therefore, ascorbate glutathione cycle might be more efficient to scavenge $\mathrm{H} 2 \mathrm{O} 2$.
\end{abstract}

Keyword: Antioxidant enzymes, Lipid peroxidase, Cultivars, Periods of stress, Rice, Submergence stress 